\title{
The Conversion and Transfer of Cannabinoids from Cannabis to Smoke Stream in Cigarettes
}

Sytze Elzinga*, Oscar Ortiz and Jeffrey C Raber

The Werc Shop, LLC, Pasadena, CA 91107, USA

\begin{abstract}
Cannabis cigarettes $(100 \%$ cannabis) with various percentages of cannabinoids were smoked through an experimental setup, and cannabinoids in the mainstream, sidestream and ash were collected. The cannabis used for the experiments predominantly contained cannabinoid acids. Less than $0.5 \%$ of the originally present tetrahydrocannabinolic acid or cannabidiolic acid was recovered as carboxylic acids in the smoke stream, indicating almost complete decarboxylation of cannabinoid acids upon combustion. Recovery of the theoretically present amount of tetrahydrocannabinol from plant material in the smoke stream varied from $27.5-46.3 \%$ and an average over all the experiments of $36.9 \%$. The recovery of the theoretically present amount of cannabidiol showed similar results with a range of recovery from $29.9-42.6 \%$, with an average of $38.4 \%$. On average over $50 \%$ of the cannabinoids originally present in the plant material could be recovered in the combined ash, mainstream and sidestream smoke. The missing mass balance is most likely caused by destruction of the cannabinoids upon combustion.
\end{abstract}

Keywords: Cannabis; Tetrahydrocannabinol; Cannabidiol; Marijuana; Cigarettes; Inhalation; Smoke stream

\section{Introduction}

Cannabidiol (CBD) and $\Delta^{9}$-tetrahydrocannabinol (THC) are the two major cannabinoids that are responsible for the primary pharmacological effects of smoked Cannabis sativa (L.).

Both THC and CBD have been extensively studied for their pharmacological effects as isolated compounds [1,2] as well as in combination [3]. Interaction between $\mathrm{CBD}$ and THC has been established; for example $\mathrm{CBD}$ has been shown to reduce the anxiety provoked by THC [4].

The primary route of administration for both medicinal and recreational use of cannabis is inhalation of the volatiles formed upon combustion or vaporization. In unheated cannabis plant matter, the cannabinoids are predominately present as their non-psychoactive carboxylic acids; cannabidiolic acid (CBDA) for CBD and $\Delta^{9}$ tetrahydrocannabinolic acid (THCA) for THC. Upon combustion of cannabis plant matter, the carboxylic acids convert into their neutral equivalents (Figure 1).

Some research has been performed on the volatilization of cannabinoids when smoked.

Mikes et al. [5], spiked less than 1 milligram of synthetic THC and CBD into tobacco cigarettes and trapped the cannabinoids in the<smiles>CCCCCc1c(C)c(C(=O)O)c(O)c2c1OC(C)(C)[C@H]1CCC(C)=C[C@H]21</smiles>

(1)

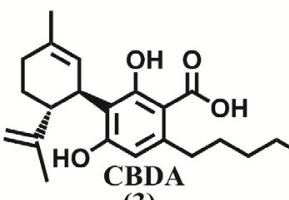

(3)

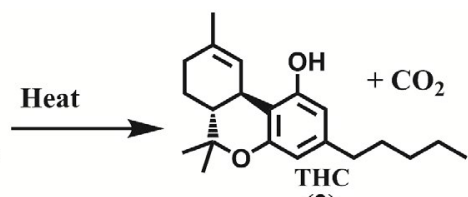

(2)

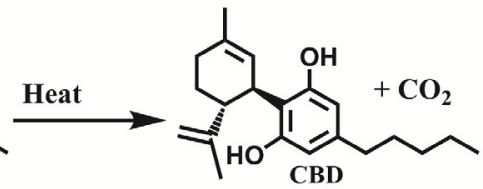

(4)
Figure 1: Decarboxylation of cannabinoid acids upon heating (1: THCA, 2 : THC, 3: CBDA, 4: CBD). smoke with a Cambridge filter. They recovered $21-23 \%$ of the spiked cannabinoids in the smoke stream and mention different ratios in the smoke than in the starting material, suggesting the partial conversion of CBD into THC. It is important to note that this study used neutral cannabinoids and not the carboxylic acid forms which are naturally present in cannabis flowers.

Davis et al. [6], studied cannabis cigarettes without tobacco. Two methods of smoking were investigated. In constant draft mode, more than $69 \%$ of the THC present in the plant material could be recovered. In the more realistic intermittent smoking regimen, $16-19 \%$ of the THC could be recovered. Two blends of cannabis plant matter were used for this study. The first blend consisted of leaves and contained 1.60\% THC, and the other consisted of both leaves and tops and contained $3.10 \%$ THC. These percentages are low compared to the current median THC level of $11.75 \%$ in cannabis seized in California in 2008 as reported by Burgdorf et al. [7].

Van der Kooy et al. reported on the effect of tobacco on the THC transfer from cannabis [8]. In this study cannabis female flowers with $17.4 \%$ THCA (equivalent to $15.3 \%$ THCmax after decarboxylation) were used. In one of the experiments, no tobacco was used in the cigarette, and $21.4 \%$ of the THCmax could be recovered in the smoke stream. Side stream smoke, cannabinoid acids, and CBD were not investigated. It was found that addition of tobacco results in a relatively higher rate of transfer of THC to the smoke stream. This effect is postulated to be caused by more efficient burning and higher burning temperatures in the presence of tobacco. Combining of tobacco and cannabis is, however, not recommended because of the health concerns associated with tobacco use.

This paper is, in our knowledge, the first to study the total mass

*Corresponding author: Dr. Jeffrey C Raber, The Werc Shop, LLC, Pasadena, CA 91107, USA, Tel: 310-703-9567; E-mail: jeff@thewercshop.com

Received November 29, 2014; Accepted December 12, 2014; Published January 06, 2015

Citation: Elzinga S, Ortiz O, Raber JC (2015) The Conversion and Transfer of Cannabinoids from Cannabis to Smoke Stream in Cigarettes. Nat Prod Chem Res 3: 163 doi:10.4172/2329-6836.1000163

Copyright: @ 2015 Elzinga S, et al. This is an open-access article distributed under the terms of the Creative Commons Attribution License, which permits unrestricted use, distribution, and reproduction in any medium, provided the original author and source are credited. 


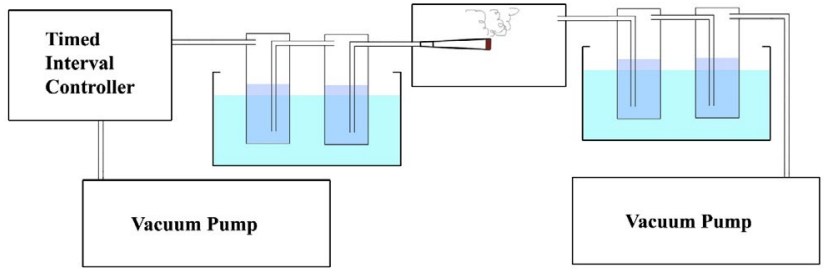

Figure 2: Experimental setup.

balance of THC, CBD, THCA and CBDA in the smoke stream, sidestream smoke and ash of a cannabis cigarette.

\section{Methods}

\section{Chemicals}

Methanol and water of analytical grade, as well as washing acetone and methanol of laboratory grade were purchased from Sigma Aldrich, St. Louis, MO, USA. Analytical standards for the cannabinoids were acquired from Restek, Bellefonte, PA, USA.

\section{Cannabis cigarettes}

Dried female cannabis flowers were ground using an IKA A11 hand held grinder acquired from IKA Works, Wilmington, NC, USA. Large stems were removed. After grinding the material was homogenized and stored for 3 weeks in a glass mason jar containing Boveda Humidity Packs, which maintained a $62 \%$ relative humidity level within the jar, to ensure uniform moisture content across all experiments. The humidity packs were acquired from Boveda, Wayzata, MN, USA. At the day of the experiment $800 \mathrm{mg}$ of the cannabis was packed into a preformed paper cone (King Size DeLuxe, $109 \times 26 \mathrm{~mm}$ filter, artnr 0100007, VanDenBerg Special Products BV, Rotterdam, The Netherlands). The built in filter consists of folded paper and leaves an open path for smoke. No tobacco was used in any of the experiments.

\section{Apparatus and method for sample collection}

In these experiments both the main stream and side stream smoke were trapped.

The experimental setup consisted of an enclosed chamber in which the cannabis cigarette was placed. A schematical representation can be found in Figure 2. The cannabis cigarette was directly pressed into a piece of tubing that was secured inside the enclosed container and led to the outside of the container, where it was connected to two gas wash bottles connected in series. A time controlled valve was connected to the exit of the second wash bottle and a vacuum pump (MD 4C, Vacuubrand, Essex, CT, USA) was connected to the timed valve.

The side stream smoke was sucked out of the enclosure through 2 separate gas wash bottles that were connected to a continually running vacuum pump (MD 4C, Vacuubrand, Essex, CT, USA).

Each gas wash bottle (4 total per experiment) contained 150 $\mathrm{ml}$ of analytical grade methanol and was chilled in a methanol bath containing dry ice. Per experiment the smoke of 2 cannabis cigarettes where collected in the same gas wash bottles. Two (2) or 3 replicates where performed per experiment. A portion of the methanol from the gas bottles was analyzed by HPLC for cannabinoid content.

The vacuum pump with a valve on an interval timer was used to simulate smoking by a human. The interval timer was programmed to open for 2 seconds at a 7 second interval. The vacuum strength was calibrated to be in the 3.5-4.6 range according to the method described in our previous publication [9].

The cigarette was lit with a lighter at the start of the experiment and the timed vacuum provided enough suction to keep the cigarette lit during experiment. Cigarettes where smoked all the way up to the filter. The total amount of ash + filter was extracted and analyzed for cannabinoid content. This value is referred to as "ash" even though it includes the filter.

\section{Analytical measurements}

Quantification of THC, CBD, THCA and CBDA was performed using a Shimadzu prominence UFLC system (Shimadzu Scientific Instruments, Columbia, MD, USA). The acidic analytical method as published by Hazekamp et al. was used [10].

\section{Calculation of THCmax and CBDmax}

Cannabinoids are present in cannabis mainly in their acidic form. Upon combustion these acidic cannabinoids decarboxylate and convert into their neutral equivalent. To be able to compare recoveries, all values are reported as the neutral equivalent.

THCmax is the sum of THC and THCA corrected for loss of $\mathrm{CO}_{2}$ This is calculated as follows:

$$
\text { THCmax }=T H C+\frac{T H C A}{358.48} * 314.47
$$

CBDmax is the sum of CBD and CBDA corrected for the loss of $\mathrm{CO}_{2}$. This is calculated as follows:

$$
C B D \max =C B D+\frac{C B D A}{358.48} * 314.47
$$

\section{Results}

\section{High THC strains}

Three (3) cannabis varieties where used for this experiment. The material was ground and equilibrated for moisture content. The day of the experiment the material was analyzed in duplicate to determine THCA, THC and THCmax content (Table 1). As expected, THCA was the dominant cannabinoid. The material contained only small amounts of CBDmax $(<0.45 \%)$ and CBN $(<0.20 \%)$.

The cigarettes contained 0.7855-0.8084 grams of cannabis each. The content weight for each individual cigarette was recorded and the absolute amount of THCmax present in the cannabis cigarettes was calculated. The total recovered amount of THC in the smoke stream, side stream and ash was calculated. Results can be found in Table 2.

The data from Table 2 was converted to show the relative proportion of the theoretical amount of THC present in the various smoke products. This data is presented in Figure 3.

On average, approximately $50 \%$ of the theoretically present amount of THC could be found in the smoke stream, side stream and ash. This

\begin{tabular}{|c|c|c|c|}
\hline Cannabis variety & THCA (w. $\%)$ & THC (w.\%) & THCmax (w. $\%)$ \\
\hline "High octane" & $13.8( \pm 0.5)$ & $1.7( \pm 0.1)$ & $13.8( \pm 0.3)$ \\
\hline "XJ-13" & $14.8( \pm 0.8)$ & $2.1( \pm 0.2)$ & $15.1( \pm 1.0)$ \\
\hline "King Louie" & $18.0( \pm 0.7)$ & $2.8( \pm 0.2)$ & $18.6( \pm 0.8)$ \\
\hline
\end{tabular}

Table 1: Cannabis material used for high THC study. (95\% confidence interval between brackets, based upon duplicate measurement). 
Citation: Elzinga S, Ortiz O, Raber JC (2015) The Conversion and Transfer of Cannabinoids from Cannabis to Smoke Stream in Cigarettes. Nat Prod Chem Res 3: 163. doi:10.4172/2329-6836.1000163

Page 3 of 5

\begin{tabular}{|c|c|c|c|c|c|}
\hline \multirow{2}{*}{ Experiment } & \multirow{2}{*}{ Replicate } & \multirow{2}{*}{$\begin{array}{c}\text { THCmax } \\
\text { in Starting Material * }\end{array}$} & \multicolumn{3}{|c|}{ THC in absolute $\mathrm{mg}$} \\
\hline & & & Mainstream & Side stream & Ash \\
\hline \multirow{3}{*}{ High Octane } & 1 & 219.8 & 101.0 & 30.9 & 14.4 \\
\hline & 2 & 221.1 & 87.4 & 18.3 & 17.1 \\
\hline & 3 & 218.6 & 81.6 & 6.7 & 14.7 \\
\hline \multirow{3}{*}{$\mathrm{XJ}-13$} & 1 & 238.8 & 64.6 & 21.2 & 13.7 \\
\hline & 2 & 240.2 & 105.7 & 29.6 & 16.1 \\
\hline & 3 & 237.7 & 87.9 & 9.0 & 19.6 \\
\hline \multirow{3}{*}{ King Louie } & 1 & 300.0 & 100.4 & 32.0 & 18.4 \\
\hline & 2 & 300.5 & 97.0 & 34.1 & 17.8 \\
\hline & 3 & 299.6 & 82.4 & 11.0 & 20.9 \\
\hline
\end{tabular}

Table 2: The recovery of THC from smoked cannabis cigarettes.

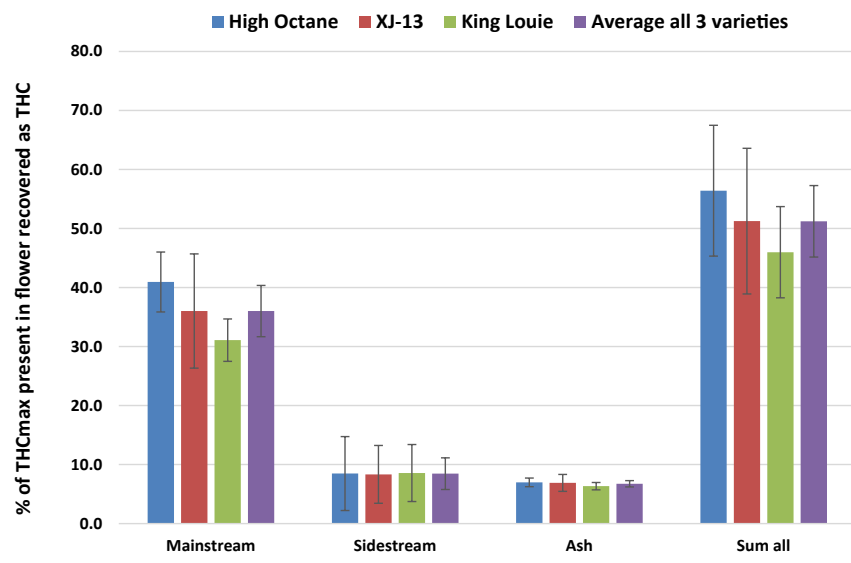

Figure 3: Recovery (\%) of THC in various smoke products (95\% confidence interval calculate from replicates indicated by error bars).

\begin{tabular}{|c|c|c|c|c|c|}
\hline \multicolumn{2}{|c|}{ Cannabinoid } & $\begin{array}{l}\text { Mainstream } \\
(\%)\end{array}$ & $\begin{array}{c}\text { Sidestream } \\
(\%)\end{array}$ & Ash (\%) & $\begin{array}{l}\text { Sum all } \\
(\%)\end{array}$ \\
\hline \multicolumn{2}{|c|}{ THCmax recovered as THC (\%) } & $36.0( \pm 4.3)$ & $8.5( \pm 2.7)$ & $6.7( \pm 0.5)$ & $51.2( \pm 6.1)$ \\
\hline \multicolumn{2}{|c|}{ THCmax recovered as THCA (\%) } & $0.3( \pm 0.1)$ & $0.0( \pm 0.0)$ & $0.4( \pm 0.2)$ & $0.7( \pm 0.2)$ \\
\hline \multicolumn{2}{|c|}{ THCmax recovered as CBN (\%) } & $0.5( \pm 0.3)$ & $0.2( \pm 0.1)$ & $0.2( \pm 0.1)$ & $1.0( \pm 0.4)$ \\
\hline \multicolumn{6}{|c|}{$\begin{array}{l}\text { Table 3: Percentage of THCmax in cannabis cigarettes that was recovered as } \\
\text { THC, THCA or CBN ( } 95 \% \text { confidence interval between brackets). }\end{array}$} \\
\hline Cannabinoid & \multicolumn{2}{|c|}{ High THC variety (w.\%) } & \multicolumn{3}{|c|}{ High CBD variety (w.\%) } \\
\hline THCA & \multicolumn{2}{|c|}{$10.9( \pm 0.1)$} & \multicolumn{3}{|c|}{$0.7( \pm 0.2)$} \\
\hline THC & \multicolumn{2}{|c|}{$2.2( \pm 0.0)$} & \multicolumn{3}{|c|}{$0.3( \pm 0.1)$} \\
\hline CBDA & \multicolumn{2}{|c|}{$0.1( \pm 0.0)$} & \multicolumn{3}{|c|}{$7.5( \pm 0.1)$} \\
\hline CBD & \multicolumn{2}{|c|}{$0.2( \pm 0.0)$} & \multicolumn{3}{|c|}{$1.0( \pm 0.0)$} \\
\hline THCmax & \multicolumn{2}{|c|}{$11.8( \pm 0.1)$} & \multicolumn{3}{|c|}{$0.9( \pm 0.3)$} \\
\hline CBDmax & \multicolumn{2}{|c|}{$0.2( \pm 0.0)$} & \multicolumn{3}{|c|}{$7.6( \pm 0.1)$} \\
\hline
\end{tabular}

Table 4: Cannabinoid content in the high THC and high CBD variety (95\% confidence interval for averages between brackets).

\begin{tabular}{|c|c|c|c|}
\hline $\begin{array}{c}\text { High THC plant } \\
\text { material in blend }\end{array}$ & $\begin{array}{c}\text { High CBD plant } \\
\text { material in blend }\end{array}$ & $\begin{array}{c}\text { THCmax in blend } \\
\text { (w.\%) }\end{array}$ & $\begin{array}{c}\text { CBDmax in } \\
\text { blend (w.\%) }\end{array}$ \\
\hline $0 \%$ & $100 \%$ & 0.9 & 7.6 \\
\hline $33 \%$ & $66 \%$ & 4.5 & 5.1 \\
\hline $50 \%$ & $50 \%$ & 6.3 & 3.9 \\
\hline $66 \%$ & $33 \%$ & 8.1 & 2.7 \\
\hline
\end{tabular}

Table 5: Cannabinoid content in the blends of high THC and high CBD variety.

raises the question of whether some of the THCA does not carboxylate and stays THCA or if THC perhaps degrades to CBN. All data was combined and Table 3 shows the average percentage of THCmax that was recovered as THC, THCA and CBN in the various combustion products.

This data shows that THCA and CBN only form in trace amounts and cannot explain the missing gap in the mass balance.

\section{Various CBD and THC blends}

Two cannabis varieties were used for this experiment. The content of the cannabinoids can be found in Table 4. Only trace amounts $(<0.1 \%)$ of $\mathrm{CBN}$ could be detected in the starting material.

It can be noticed that most of the cannabinoids are present in their carboxylic acid form.

The high CBD variety was blended with the high THC variety in various ratios. The theoretical amounts of THCmax and CBDmax in these blends can be found in Table 5 .

Per experiment, 2 cigarettes containing an exactly known amount of approximately $800 \mathrm{mg}$ of blended cannabis flower were smoked. Two (2) experiments per blend ratio were performed resulting in 8 analytical data points.

Recoveries for CBD and THC in the various experiments can be found in Table 6 .

Visual inspection of the data in Table 6 did not indicate any correlation between the blend ratios and amounts of THC or CBD recovered in the smoke stream. The data in Table 6 was therefore pooled and the recovery of CBD was compared with the recovery of THC in the various combustion products. An F-test was performed and showed equal variance in both groups. A 2 tailed T-test was performed and showed no significant difference with $95 \%$ certainty between the recoveries of CBD compared to the recovery of THC in either smoke stream, sidestream, ash or overall recovery, indicating a similar behavior upon combustion. For this reason the results for the various blends were averaged and the $95 \%$ confidence interval is calculated. This was also performed for the cannabinoid acids and CBN. These results can be found in Table 7.

The quantity of THCmax and CBDmax in the individual cannabis cigarettes were calculated and the absolute amounts of THC and CBD in the smoke stream were analyzed. Using this data, ratios of THC/ CBD were determined in the cannabis cigarette and smoke stream. Figure 4 shows the graph when this data is plotted. A good correlation $\left(\mathrm{R}^{2}=0.9948\right)$ between the ratio of THC/CBD in cannabis cigarettes and resulting smoke was observed.

\section{Discussion and Conclusions}

In this study, the transfer of cannabinoids from plant material to smoke stream was investigated.Investigated ranges in the plant material 
Citation: Elzinga S, Ortiz O, Raber JC (2015) The Conversion and Transfer of Cannabinoids from Cannabis to Smoke Stream in Cigarettes. Nat Prod Chem Res 3: 163. doi:10.4172/2329-6836.1000163

Page 4 of 5

\begin{tabular}{|c|c|c|c|c|c|c|}
\hline \multicolumn{7}{|c|}{ Recovery of CBDmax present in plant material as CBD (\%) in combustion products } \\
\hline High THC plant & High CBD plant & \multirow{2}{*}{ Replicate } & Smoke & Side & \multirow{2}{*}{ Ash } & \multirow{2}{*}{ Overall } \\
\hline material in blend & material in blend & & Stream & Stream & & \\
\hline $0 \%$ & $100 \%$ & 1 & 42.6 & 9.5 & 3.6 & 55.8 \\
\hline $0 \%$ & $100 \%$ & 2 & 42.2 & 9.0 & 4.4 & 55.6 \\
\hline $33 \%$ & $66 \%$ & 1 & 40.5 & 3.9 & 3.6 & 47.9 \\
\hline $33 \%$ & $66 \%$ & 2 & 38.5 & 13.0 & 4.8 & 56.4 \\
\hline $50 \%$ & $50 \%$ & 1 & 35.0 & 8.6 & 2.9 & 46.5 \\
\hline $50 \%$ & $50 \%$ & 2 & 42.1 & 12.8 & 5.0 & 59.9 \\
\hline $66 \%$ & $33 \%$ & 1 & 36.6 & 14.3 & 3.5 & 54.4 \\
\hline $66 \%$ & $33 \%$ & 2 & 29.9 & 16.5 & 4.6 & 51.1 \\
\hline \multicolumn{7}{|c|}{ Recovery of THCmax present in plant material as THC (\%) } \\
\hline High THC plant & High CBD plant & \multirow{2}{*}{ Replicate } & Smoke & Side & \multirow{2}{*}{ Ash } & \multirow{2}{*}{ Overall } \\
\hline material in blend & material in blend & & Stream & Stream & & \\
\hline $0 \%$ & $100 \%$ & 1 & 31.0 & 7.5 & 3.0 & 41.4 \\
\hline $0 \%$ & $100 \%$ & 2 & 34.8 & 7.3 & 3.9 & 46.0 \\
\hline $33 \%$ & $66 \%$ & 1 & 40.2 & 5.1 & 3.5 & 48.9 \\
\hline $33 \%$ & $66 \%$ & 2 & 39.6 & 13.2 & 4.6 & 57.4 \\
\hline $50 \%$ & $50 \%$ & 1 & 33.8 & 10.3 & 2.8 & 46.9 \\
\hline $50 \%$ & $50 \%$ & 2 & 44.3 & 14.5 & 5.4 & 64.2 \\
\hline $66 \%$ & $33 \%$ & 1 & 39.7 & 14.1 & 4.4 & 58.1 \\
\hline $66 \%$ & $33 \%$ & 2 & 29.5 & 7.5 & 6.1 & 43.1 \\
\hline
\end{tabular}

Table 6: Recoveries of $\mathrm{CBD}$ and $\mathrm{THC}$ in the various combustion products.

\begin{tabular}{|c|c|c|c|c|}
\hline Cannabinoid & Mainstream smoke & Sidestream smoke & Ash & Overall Sum \\
\hline THCmax Recovered as THC (\%) & $36.6( \pm 3.6)$ & $9.9( \pm 2.5)$ & $4.2( \pm 0.8)$ & $50.8( \pm 5.7)$ \\
\hline THCmax recovered as THCA (\%) & $0.3( \pm 0.1)$ & $N D( \pm N D)$ & $0.1( \pm 0.1)$ & $0.4( \pm 0.2)$ \\
\hline THCmax recovered as CBN (\%) & $0.4( \pm 0.3)$ & $0.2( \pm 0.2)$ & $0.1( \pm 0.1)$ & $0.8( \pm 0.4)$ \\
\hline CBDmax recovered as CBD (\%) & $38.4( \pm 3.1)$ & $11.0( \pm 2.8)$ & $4.1( \pm 0.5)$ & $53.4( \pm 3.2)$ \\
\hline CBDmax recovered as CBDA (\%) & $0.4( \pm 0.1)$ & $0.1( \pm 0.1)$ & $0.1( \pm 0.1)$ & $0.5( \pm 0.1)$ \\
\hline
\end{tabular}

Table 7: Average recovery of cannabinoids in various combustion products ( $95 \%$ confidence interval for averages between brackets).

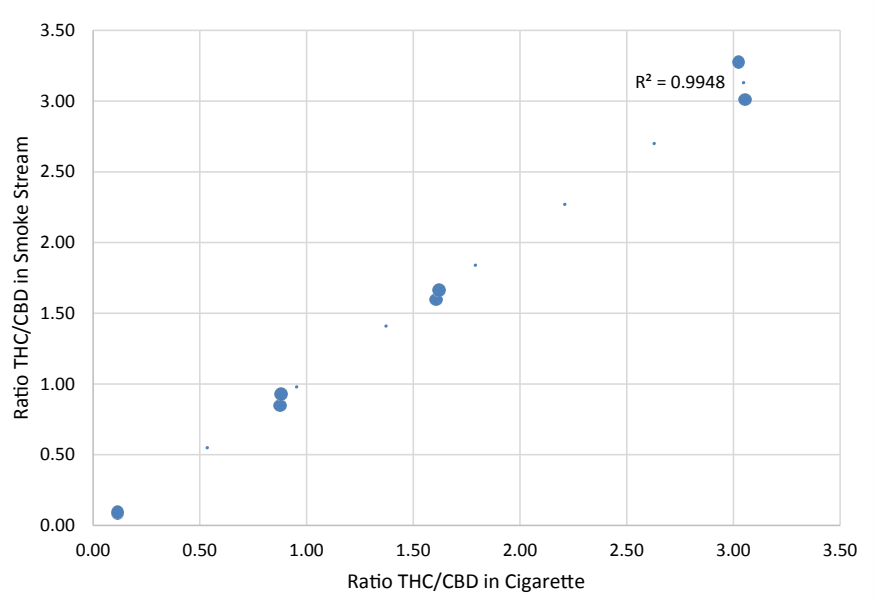

Figure 4: Correlation between ratio THC/CBD in cigarette and smoke stream.

were $4.5-18.6 \%$ THCmax and $2.7-7.6 \%$ for CBDmax. All cannabis cigarettes contained only cannabis, contrary to some previous studies in which cannabis was blended with tobacco.

Although the plant material used for this experiment contained predominantly cannabinoid acids, mainly neutral cannabinoids were recovered. Less than $0.5 \%$ of the originally present THCmax or CBDmax was recovered as carboxylic acids in the mainstream smoke, sidestream smoke and ash. This indicates almost complete decarboxylation of the cannabinoid acids during cannabis smoking.
Recovery of THCmax as THC in the smoke stream varied from 27.5 to $46.3 \%$ with an average of $36.9 \%$ over all the experiments. The recovery of CBDmax as CBD showed similar results with a range of recovery from $29.9-42.6 \%$ with an average of $38.4 \%$. There was no statistically $(\mathrm{p}=0.05)$ significant difference between the CBD and THC recoveries. The recoveries in the smoke stream are higher than previously reported levels, which were in the $16-23 \%$ range $[5,6,8]$. We expect this is the result of differences in the plant material, smoking protocol and collection methods.

No conversion of CBD to THC was observed in this experiment as indicated by similar recoveries for THCmax in the presence and absence of CBD as well as the consistency of ratios in cigarette versus smoke in different blends. This is in conflict with the results reported by Mikes et al., which suggest THC might form from CBD upon smoking [5]. It could be that this effect only occurs in tobacco-based cigarettes, as tobacco smoke is significantly more acidic than marijuana smoke [11], and acidic conditions are known to convert CBD to THC [12].

We showed that THC/CBD ratios remained constant upon combustion. This indicates that specific ratios of THC to CBD can be attained by blending a high THC with a high CBD variety. This could be of importance for future studies in which the interaction between THC and CBD is investigated.

On average over all of the experiments, $9.2 \%$ of the THCmax could be recovered as THC in the sidestream smoke and 5.6\% could be found in the ashes. For CBDmax $11.0 \%$ could be recovered in the side stream smoke and $4.1 \%$ in the ash. When variations between replicates are considered, there is no significant difference between these values. This indicates a similar behavior for THC and CBD. 
On average a little over $50 \%$ of the THCmax and CBDmax present in the plant material could be recovered in mainstream smoke, sidestream smoke and ash. Experiments with a third inline trap on either the smoke stream or sidestream side indicated that $>90 \%$ of the cannabinoids got trapped in the first two gas traps (data not shown) and therefore the missing mass balance cannot be explained by incomplete trapping. A minimal amount of tubing was used and recovery experiments in which we extracted the tubing revealed only trace amounts of cannabinoids. The walls of the enclosure have also been tested, but the cannabinoids present did not influence the mass balance gap significantly. The HPLC chromatograms did not reveal any major unknown peaks that would be able to explain the gap in the mass balance either. This leads us to conclude that the missing cannabinoids have been degraded by combustion to some unknown compound(s).

This data demonstrates that doctors and patients can utilize blended cannabis material to provide different dosing regimens for inhalation that can be tailored to meet each individual patient's needs even allowing for cannabinoid ratio combinations that may not be present in nature.

\section{References}

1. Mechoulam R, Peters M, Murillo-Rodriguez E, Hanus LO (2007) Cannabidiol-recent advances. Chem Biodivers 4: 1678-1692.

2. Costa B (2007) On the pharmacological properties of Delta9tetrahydrocannabinol (THC). Chem Biodivers 4: 1664-1677.
3. Russo E, Guy GW (2006) A tale of two cannabinoids: the therapeutic rationale for combining tetrahydrocannabinol and cannabidiol. Med Hypotheses 66: 234246.

4. Zuardi AW, Shirakawa I, Finkelfarb E, Karniol IG (1982) Action of cannabidio on the anxiety and other effects produced by delta 9-THC in normal subjects. Psychopharmacology (Berl) 76: 245-250.

5. Mikes F, Waser PG (1971) Marihuana components: effects of smoking on delta9-tetrahydrocannabinol and cannabidiol. Science 172: 1158-1159.

6. Davis KH, McDaniel IA, Caddell LW, Moody PL (1984) Some smoking characteristics of marijuana cigarettes. In: Agurell S, Dewey WL, Willette RE et al (1984) The Cannabinoids: Chemical, Pharmacologic, and Therapeutic Aspects 97-109.

7. Burgdorf JR, Kilmer B, Pacula RL (2011) Heterogeneity in the composition of marijuana seized in California. Drug Alcohol Depend 117: 59-61.

8. Van der Kooy F, Pomahacova B, Verpoorte R (2009) Cannabis smoke condensate II: influence of tobacco on tetrahydrocannabinol levels. Inhal Toxicol 21: 87-90.

9. Sullivan N, Elzinga S, Raber JC (2013) Determination of pesticide residues in cannabis smoke. J Toxicol 2013: 378168.

10. Hazekamp A, Peltenburg A, Verpoorte R, Giroud C (2005) Chromatographic and Spectroscopic Data of Cannabinoids from Cannabis sativa $L$. Journal of Liquid Chromatography \& Related Technologies 28: 2361-2382.

11. Moir D, Rickert WS, Levasseur G, Larose Y, Maertens R, et al. (2008) A comparison of mainstream and sidestream marijuana and tobacco cigarette smoke produced under two machine smoking conditions. Chem Res Toxicol 21: 494-502.

12. Adams R, Cain CK, McPhee WD, Wearn RB (1941) Structure of Cannabidiol. XII. Isomerization to Tetrahydrocannabinols. J Am Chem Soc 63: 2209-2213. 